P92 WHAT IS THE BURDEN OF ASPERGILLOSIS AND OTHER OPPORTUNISTIC FUNGAL INFECTIONS IN PATIENTS WITH SEVERE INFLUENZA AND COVID-19 IN THE ICU?

1J Menendez Lorenzo, ${ }^{1,2}$ DJ Dhasmana. ${ }^{1}$ University of St Andrews, St Andrews, UK; ${ }^{2}$ NHS Fife, Kirkcaldy, UK

\subsection{6/thorax-2021-BTSabstracts.202}

Background Co-infection with Aspergillus previously described to cause significant morbidity and mortality in those with severe Influenza, has more recently been described in COVID19. 'Influenza-Associated Pulmonary Aspergillosis' (IAPA) and 'COVID-Associated Pulmonary Aspergillosis' (CAPA) have been reported in up to $23 \%$ and $35 \%$ of severe disease, respectively. Establishing evidence of invasive Aspergillosis (IA) in these patients is challenging, requiring specific clinical, radiological and microbiological criteria. The burden of IAPA and CAPA in the ICU in our region is unknown.

Aims To identify the incidence of invasive Aspergillosis (IA) and other opportunistic fungal infection in those with severe Influenza and COVID-19 in a district general hospital, Fife, Scotland.

Methods Retrospective cohort review of ICU admissions with severe Influenza or COVID-19 from May 2017 - February 2021. IA was diagnosed using international definitions according to EORTC/MSG, AspICU and modified AspICU criteria. Results 89 patients were identified with Influenza (27; median age 53.3 yrs, male 56\%) and COVID-19 (62; median age 59.1 yrs, male 61\%). No case satisfied criteria for definite IA, however, the majority of patients did not undergo all relevant tests; CT imaging features in 26/89 (29.2\%), and fungal biomarkers in 3/89 (3.4\%). Two patients demonstrated Aspergillus culture from respiratory samples but did not meet other criteria. Fungal infections were identified in 39/89 (44\%), the majority Candida (37), mostly from ET secretions (54\%). Candida was significantly higher in COVID-19 than in Influenza, including 2 patients with Candidaemia. Positive fungal culture was associated with increased length of stay (43d vs $20 \mathrm{~d}$ ), ICU bed days (26d vs $19 \mathrm{~d}$ ), but not mortality (33.3\% vs $30.0 \%)$. Few patients $(7.9 \%)$ received antifungal treatment, with possible explanations including unclear diagnosis, high costs, uncertain benefit. 54/89 (60.7\%) demonstrated bacterial co-infection, including $31 / 89 \quad(34.8 \%)$ with bacteraemia (COVID, 23; Influenza, 8).

Conclusions IAPA and CAPA were not identified in this 4-year cohort, although case finding was limited by inadequate diagnostics. Timely access to fungal biomarkers compromises diagnostic testing. The incidence is likely to be low, despite the significant study limitations. We recommend prospective systematic practice of investigations and improved fungal diagnostics to better understand the burden of Aspergillosis in these patients.

\section{P93 COMPARISON OF INFLAMMATORY PROFILES BETWEEN COVID-19 AND OTHER ACUTE LOWER RESPIRATORY TRACT INFECTIONS: RESULTS FROM THE PREDICT- COVID19 STUDY} ${ }^{1} \mathrm{~A}$ Gilmour, ${ }^{1} \mathrm{C}$ Hocking, ${ }^{1} \mathrm{BJM}$ New, ${ }^{1} \mathrm{D}$ Connell, ${ }^{1} \mathrm{H}$ Richardson, ${ }^{1} \mathrm{D}$ Cassidy, ${ }^{1} \mathrm{~A}$ Shoemark ${ }^{1} \mathrm{JD}$ Chalmers. 'Scottish Centre for Respiratory Research, University of Dundee, Dundee, UK 2University of Sheffield, Sheffield, UK

10.1136/thorax-2021-BTSabstracts.203
Introduction COVID-19 has been reported to induce a 'cytokine storm' distinct from other acute respiratory tract infections (LRTIs). Understanding the similarities and differences in inflammatory profiles between SARS-CoV-2 infection and other respiratory infections may aid diagnosis, as well as the potential to repurpose therapies such as steroids and anti-IL-6 receptor antagonists for other respiratory infections.

Methods A prospective observational study of patients in 3 groups 1) PCR confirmed SARS-CoV-2 infection, 2) community-acquired pneumonia (CAP) without SARS-CoV-2, and 3) controls hospitalized for reasons other than infection. Patients were enrolled from a single centre in Dundee, UK. Patients were enrolled within 96 hours of hospital admission. 45 inflammatory biomarkers were measured in blood using the Olink target proteomic based biomarker panel. Additional markers were measured by ELISA/immunoassay and enzyme activity assay as appropriate. Discrimination between groups was evaluated using the area under the receiver operator characteristic curve (AUC).

Results 294 patients were included (COVID-19 $\mathrm{n}=176$, CAP $\mathrm{n}=76$, controls $\mathrm{n}=42)$, mean age $64(\mathrm{SD} \pm 15.2)$ and 150 subjects were male (51.0\%). Using ROC analysis the most discriminating biomarkers for COVID-19 compared to CAP were CXCL-10 (AUC 0.84 95\%CI $0.78-0.90 \quad \mathrm{p}<0.001$ ), CCL-8 $(0.87$ 95\%CI $0.82-0.92, \quad \mathrm{p}<0.001), \quad \mathrm{CCL}-7 \quad(0.84 \quad 95 \% \mathrm{CI}$ 0.78-0.89, p<0.001), CXCL-11 (0.80 95\%CI 0.73-0.88, $\mathrm{p}<0.001)$. Further biomarkers included IL-18, IL-7, IL-10 and IL-33. The most discriminating biomarkers for COVID-19 compared to controls were CXCL-10 (0.89 95\%CI 0.85-0.93, $\mathrm{p}<0.001$, CCL-7 (0.88 95\%CI 0.83-0.92, p<0.001), CCL-8 $(0.8795 \%$ CI $0.82-0.92, \quad \mathrm{p}<0.001)$. Further biomarkers included IL-10, CXCL-11 and IL-18. IL-4 was significantly lower in COVID-19 patients compared to controls $(0.2795 \%$ CI $0.16-0.38, \mathrm{p}<0.001)$. No significant difference in IL-6 was seen between COVID-19 and CAP (median $21.9 \mathrm{pg} / \mathrm{ml}$ vs $19.8 \mathrm{pg} / \mathrm{ml}, \mathrm{p}=0.59)$.

Conclusion Differential markers of inflammation were identified between COVID-19, CAP and control samples, indicating distinct immunological pathways. The identification of a similar IL-6 signature between COVID-19 and CAP indicates that IL-6 targeting therapies currently being used to treat COIVD19 may also be beneficial in the treatment of CAP.

\section{P94 INFLUENZA AND COVID-19 PNEUMONIA: THE DIFFERENCE IS PULMONARY HYPERTENSION}

S Desai, A Devaraj, S Dintakurti, C Mahon, S Padley, S Singh, B Rawal, C Ridge, T Semple. Royal Brompton Hospital, London, UK

\subsection{6/thorax-2021-BTSabstracts.204}

Purpose To describe the incidence of pulmonary artery thrombosis in COVID-19 versus influenza pneumonia using CT angiography and to assess whether it may increase the risk of pulmonary hypertension.

Materials and Methods Single and dual energy CT pulmonary angiography of age- and gender-matched patients with influenza and COVID-19 pneumonia, referred for extra-corporeal membrane oxygenation (ECMO) and/or mechanical ventilation from January 2016 to January 2021, were retrospectively evaluated. Two independent observers qualitatively and quantitively assessed clot burden and Qanadli CT Obstruction Index. Two consensus observers calculated pulmonary artery volume 